\title{
Constitutive and Inducible Innate Responses in Cells Infected by HSV-1- Derived Amplicon Vectors
}

\author{
Eliza Tsitoura and Alberto L. Epstein*
}

\author{
Université de Lyon, Lyon, F-69003, France; CNRS, UMR5534, Centre de Génétique Moléculaire et Cellulaire, \\ Villeurbanne, F-69622, France
}

\begin{abstract}
Amplicons are helper-dependent herpes simplex virus type 1 (HSV-1)-based vectors that can deliver very large foreign DNA sequences and, as such, are good candidates both for gene delivery and vaccine development. However, many studies have shown that innate constitutive or induced cellular responses, elicited or activated by the entry of HSV-1 particles, can play a significant role in the control of transgenic expression and in the induction of inflammatory responses. Moreover, transgene expression from helper-free amplicon stocks is often weak and transient, depending on the particular type of infected cells, suggesting that cellular responses could be also responsible for the silencing of amplicon-mediated transgene expression. This review summarizes the current experimental evidence underlying these latter concepts, focusing on the impact on transgene expression of very-early interactions between amplicon particles and the infected cells, and speculates on possible ways to counteract the cellular protective mechanisms, thus allowing stable transgene expression without enhancement of vector toxicity.
\end{abstract}

Keywords: HSV-1, amplicon vectors, innate responses, IFN, gene silencing.

\section{INTRODUCTION}

Pathogenic viruses and their hosts coevolved during millions of years and have developed adaptive features that allow the survival of both. The vast number and outstanding complexity of self-protective mechanisms is a clear illustration of the strength of the selective pressures that viruses and their hosts have imposed to each other. The number of genes involved in the setting up and management of the two arms of the adaptive immune system, as well as in the signalling, coordination, and execution of non-specific inducible innate mechanisms, such as the interferon (IFN), the inflammatory, and the pro-apoptotic responses, is overwhelming. The sophistication of other mechanisms, such as autophagy, the complement cascade, or the more recently recognized set of functions that can act as constitutive intracellular barriers through the silencing of viral gene expression, all concur to demonstrate that a significant fraction of the host genome is devoted to these selfprotective activities.

The same consideration is valid for viruses, at least for some of them. Viruses have evolved complex phenotypes that can be thought as operating to mask their presence within the organism or within the cells, such as latency or any other form of controlled and reversible gene silencing, integration of their genomes within cellular chromosomes, expression of non-immunogenic proteins, and disruption or diversion of the host defences. The end-result of this struggle is that, while the hosts generally prevail at the level of the whole organism, thus resulting in host survival, lytic viruses

*Address correspondence to this author at the Centre de Génétique Moléculaire et Cellulaire CGMC - UMR 5534 - CNRS Université Claude Bernard Lyon 1 Bâtiment Grégoire Mendel, 16 rue Raphaël Dubois, 69622 Villeurbanne Cedex, France; Tel: (33) 4724313 25; Fax: (33) 4724326 85; E-mail: epstein@cgmc.univ-lyon1.fr most often gain the battle at the cellular level, thus warranting virus survival and dissemination. However, viruses have small genomes and express a limited amount of proteins, suggesting that they can act only on a few key elements of the host responses and should gather together several functions into a single protein.

In the case of large nuclear DNA viruses, such as herpes simplex virus type 1 (HSV-1) the number of genes that are devoted to the virus self-protective activities is quite large, representing some $15 \%$ of the virus encoded proteins. As illustrated in Table 1, HSV-1 has developed functions able to efficiently counteract most of the above mentioned host defences. Some HSV-1 proteins, such as US3, ICP0, and ICP34.5, can supply more than one activity and play more than one role in supporting virus multiplication [1-6]. Other proteins, such as vhs (UL41) and ICP27 (UL54), probably act through non-specific mechanisms, as both of them are independently able to down-regulate expression of most cellular proteins $[7,8]$. Other proteins, such as ICP47 (UL12), gD, gC, gJ, gE, or US11, seem to play specific roles in counteracting more defined cellular responses [9-15]. Another virus protein, known as ICP4, has also been involved in escaping cellular responses, but it is not yet clear whether there is a direct involvement of ICP4 or if its contribution stems from the fact that ICP4 is a regulatory protein required for the expression of other classes of virus proteins [16]. Table 1 presents a summary of the cellular protective mechanisms acting against HSV-1 infection as well as the HSV-1 functions that are involved in counteracting the host responses.

It should be stressed, however, that the ability to counteract host responses is a feature only of wild type virus. Viral vectors, in contrast, are non-natural entities that have been developed with the aim to deliver genes for therapeutic interventions or as tools for fundamental studies, and these 
Table 1. Host Defence Mechanisms and HSV-1 Genes Involved in Disarming Cellular Responses

\begin{tabular}{|c|c|c|}
\hline Host Defence & Virus gene Involved in Counteraction & Mechanisms \\
\hline Complement cascade & $\begin{array}{l}\mathrm{gC} \text { (UL44) } \\
\mathrm{gE} \text { (US7) }\end{array}$ & $\begin{array}{l}\text { Binds C } 3 b[14,52] \\
\text { Inhibits } \mathrm{C} 5 \text { and factor } \mathrm{P} \text { (properdin) binding [15] } \\
\text { Fc receptor activity [53] }\end{array}$ \\
\hline $\begin{array}{l}\text { Intrinsic } \\
\text { innate responses }\end{array}$ & ICP0 (RL2) & E3-Ubiquitin-ligase. Induces proteolysis of transcriptional silencers [41-45] \\
\hline $\begin{array}{l}\text { Inducible } \\
\text { innate responses } \\
\text { a) Interferons } \\
\text { b) Inflammation }\end{array}$ & $\begin{array}{l}\text { ICP0 (RL2) } \\
\text { ICP27 (UL54) } \\
\text { ICP34.5 (RL1) } \\
\text { US11 } \\
\text { Vhs (UL41) } \\
\text { US3 }\end{array}$ & $\begin{array}{l}\text { Inhibition of IRF3 signalling }[3,4,54-56] \\
\text { Blocks splicing. Inhibits activation of IRF3 and STAT1 }[8,57] \\
\text { Dephosphorylates eIF2 } \alpha \text {. Prevents IRF3 activation }[3,5,12,58,59] \\
\text { Interacts with PKR, prevents production of 2'-5' OAS }[12,60,61] \\
\text { Inhibits ISG transcription and STAT1 phosphorylation. Induces degradation of } \\
\text { cellular mRNA [7, 54, 62] } \\
\text { Post-translational modification of IFN receptors and inhibition of ISG induction } \\
{[2,63]}\end{array}$ \\
\hline $\begin{array}{l}\text { Inducible } \\
\text { innate responses } \\
\text { a) Apoptosis }\end{array}$ & $\begin{array}{l}\text { US3 } \\
\text { ICP4 (RS1) } \\
\text { ICP27 (UL54) } \\
\text { gD (US6) } \\
\text { gJ (US5) } \\
\text { LAT }\end{array}$ & $\begin{array}{l}\text { Activates PKA and phosphorylates the same targets as PKA (Bad, Bid, procaspase } \\
\text { 3) }[1,64-67] \\
\text { Regulatory protein }[16,68,69] \text {. } \\
\text { Activates NFKB and stabilizes Bcl2 }[70,71] \\
\text { Activates NFKB. Protection against FAS-mediated apoptosis }[11,72] \\
\text { Prevents apoptosis induced by UV, anti-FAS or CTL killing }[10,11] \\
\text { Inhibits apoptosis during HSV-1 latency }[73,74]\end{array}$ \\
\hline Autophagy & ICP34.5 (RL1) & Binding and inhibition of Beclin 1 (Atg-6) [6] \\
\hline $\begin{array}{l}\text { Adaptive } \\
\text { immune response }\end{array}$ & $\begin{array}{l}\mathrm{gE} \text { (US7) } \\
\text { ICP47 (UL12) }\end{array}$ & $\begin{array}{l}\text { Fc receptor activity [13] } \\
\text { Binding and inhibition of human TAP [9] }\end{array}$ \\
\hline
\end{tabular}

vectors should be safe, both for the whole organism and for the infected cells. In order to decrease virus toxicity and preserve the transduced cells, one or more viral genes are often deleted or inactivated, therefore resulting in the direct or indirect silencing of genes that are involved in virus survival in the cellular environment [17]. As a consequence, the infected cells will also succeed in the struggle at the cellular level, and the vector genome, together with the therapeutic transgenes, will be silenced or eliminated [18], which constitutes a major hurdle for the use of defective vectors in gene therapy approaches.

High-capacity vectors, such as HSV-1-derived amplicon vectors $[19,20]$, are extreme cases since they carry no virus genes and therefore express no virus functions in the transduced cells. This implies that all virus self-protective functions are absent, with the only exception of the functions carried by the structural proteins that are introduced in the cells during entry of vector particles, which have nevertheless a limited half-life and will soon disappear. This is probably at the basis of the low or transient expression often observed with helper-free amplicon vectors, both in cultured cells and in experimental animals [21, 22]. Actually, many observations suggest that expression from helper-free amplicons is transient in many experimental settings and that the length and intensity of transgene expression is cell-type specific. Indeed, after infection of dividing cells, the loss of the amplicon genomes is fast since there is no vector amplification in the infected cells and the genome will therefore be diluted concomitantly with cell proliferation. However, also in cells that do not divide the amplicon genome is often expressed during a relatively short time, and this is not related to cell death. Therefore, the amplicon genome probably becomes silenced. There are no complete answers yet to explain why transgenic expression from an amplicon genome becomes extinct in the absence of helper proteins. Nevertheless, at least two non-mutually exclusive hypotheses may be advanced to explain failure to obtain stable and strong transgene expression from the amplicon genome. These are based either (i) on the induction of innate immune responses able to repress transgene expression, or (ii) on the action of constitutive cellular functions able to induce transcriptional silencing of the disabled vector genome. This review will focus on the evidences supporting these cellular responses, and will consider some possible ways to counteract these mechanisms without enhancing vector toxicity.

\section{AMPLICON VECTORS}

Amplicons are defective, helper-dependent vectors that carry no viral genes and take advantage of the large carrier capacity of the virus particle to deliver long transgenic sequences $[19,20]$. There is currently no other vector that equals the ability of amplicons to safely deliver almost 150 $\mathrm{Kbp}$ of foreign DNA to the nucleus of mammalian cells.

The genome of amplicon vectors derives from a plasmid, named the amplicon plasmid, which carries one packaging signal and one origin of DNA replication from HSV-1. As it carries no virus genes, this plasmid requires the presence of a helper virus genome to express the proteins necessary for its amplification and packaging into HSV-1 particles. Over the last decade, technological improvements have enabled the production of relatively large amounts of amplicon stocks 
that are, not or only, minimally contaminated with helper virus particles [23-26], and to use these vectors to deliver long genomic sequences to target cells [27-30]. These major breakthroughs have made it possible to study important aspects of the biological features of amplicons in the absence of helper HSV-1. However, our understanding of amplicon biology remains fragmentary in many aspects.

In addition to their applications as gene delivery vectors, amplicons are powerful tools to investigate HSV-1 functions [31-33]. Since amplicons carry no virus genes, they can be used to study very early interactions between HSV-1 particles and host cells, including the innate cellular responses elicited by virus entry, thus avoiding the requirement to use virus mutants, chemicals, or UVinactivation of virus particles [34]. UV-treatment of the virus may disrupt the protein structure of the particles affecting entry and may crosslink viral proteins and nucleic acids affecting nuclear release of the genome. Amplicons, in contrast, are able to normally penetrate cells, target their genome to the nucleus and are transcriptionally active. Furthermore, the amplicon genome can be engineered to express selected viral or cellular proteins potentially affecting early virus host interactions, thereby allowing analyzing how these functions modulate the cellular responses [21]. Since the presence of proteins expressed from helper particles in helper-contaminated (HC)-amplicon stocks can affect induction of cellular responses, in the following paragraphs we will focus mainly on studies conducted with helper-free (HF)-amplicon stocks, or with amplicon stocks only barely contaminated (less than $1 \%$ ) with defective helper particles.

\section{INDUCTION OF INNATE RESPONSES IN AMPLICON-INFECTED CELLS}

Only a few studies have addressed the cellular responses elicited by HF-amplicons. In an early study, designed to develop an immunotherapeutic approach for chronic lymphocytic leukemia (CLL), Tolba et al. compared the ability of HF-amplicons and HC-amplicons, expressing CD80 (B7.1) or CD154 (CD40L), to transduce human CLL $B$ cells and to induce immune responses [35]. Results from this study indicated that although both HF and $\mathrm{HC}$ amplicon stocks were equivalent in their ability to transduce CLL cells, a robust T-cell proliferative response was observed only with the HF-amplicons. The authors concluded that HFamplicons were better suited for immunotherapy than HCvectors and that a strong immunosuppressive activity was associated with the helper particles present in the HCamplicon stocks [35]. In a more recent study, and using a similar approach, the same group identified the viral ICP0 protein, which is expressed only in the HC-amplicon stocks, as an inhibitor of the TLR-mediated inflammation. Further, they showed that ICP0 is able to block NF- $\mathrm{KB}$ and JNK activation downstream of TLR signal activation and that this process depends on ICP0-mediated translocation of the deubiquinating enzyme USP7 from the nucleus to the cytoplasm, where this enzyme binds to and deubiquitinates TRAF6 and IKK $\gamma$, thus terminating the TLR response [36].

HF-amplicons can induce a significant inflammatory response also in the mouse brain, but this response is considerably lower than that observed using $\mathrm{HC}$-amplicon stocks [37]. In one study, C57BL/6 mice were stereotactically inoculated with $\beta$-galactosidase expressing amplicons, either contaminated or not with HSV-1 helper particles. After killing the mice, at 1 or 5 days posttransduction, samples were analysed for various cytokine, chemokine, and adhesion molecule gene expression using immune-cytochemistry and RT-PCR. Results indicated that both vector stocks induced inflammation, with blood-brain barrier opening, on day 1 . By day 5, mRNA levels of the inflammatory cytokines IL- $1 \beta$, TNF $\alpha$, or IFN $\gamma$, chemokines, such as MCP1 and IP-10, and adhesion molecule ICAM1, had returned to baseline in saline injected mice and to near baseline in animals injected with HF-amplicon stocks. In contrast, mice inoculated with HC-amplicon stocks showed elevated inflammatory molecule expression and immune cell infiltration even at day 5 post-injection. This study therefore confirmed that, although contaminating viral proteins could play a major role in the induction of inflammation in the brain, HF-amplicon vectors did induce cellular inflammatory responses in the infected host.

Other studies have focused on the IFN responses elicited by amplicons $[38,39]$. A first investigation showed that, after systemic delivery of HF-amplicon vectors into mice, early activation of the signal transducer and activator of transcription 1 (STAT1) transcription factor, a key IFNactivated signalling molecule, suppresses transcription of the vector-encoded transgene (luciferase) in the liver [38]. A similar experiment conducted in STAT1-knockout mice showed 10-fold higher luciferase expression than in wildtype mice, and this expression remained detectable during at least 80 days, while in wild-type mice luciferase expression became undetectable after 2 weeks post-infection. Additional studies using fibroblasts derived from wild-type and STAT1knockout mice revealed the significance of STAT1 signalling in transcriptional silencing of the ampliconencoded transgene in cultured cells, indicating that type I IFN induced by systemic delivery of amplicons may initiate a cascade of immune responses eventually able to suppress transgene expression at the transcriptional level. In a further study by the same group, antiviral responses were investigated following stereotactic HF-amplicon administration into the mouse striatum [39]. In this area of the brain, induction of type I IFN was rather modest and luciferase expression lasted over a year, despite dosedependent inflammation and infiltration of immune cells around the injection sites. These findings indicate that the spectrum of host responses can differ significantly depending on target organs and administration routes.

More recently, it was shown that GFP-expressing amplicon infection of cultured human foetal foreskin fibroblasts (HFFF-2) resulted in the induction of an interferon regulatory factor 3 (IRF3)-dependent antiviral response [34]. This innate response is characterized by the up-regulation of IRF7 and Toll-like receptors 3 and 4 (TLR3/TLR4), the up-regulation of some interferon stimulated genes (ISG), such as ISG54 and ISG56, and the secretion of low levels of $\beta$-IFN. These responses led to the establishment of an antiviral state in the amplicon-infected cells, which become refractory to subsequent infection with vesicular stomatitis virus (VSV). Furthermore, amplicon infection induced the nuclear translocation and subsequent degradation of IRF3, but hyperphosphorylation of this 
protein was not observed. Knockdown of IRF3 and IRF7 using specific siRNA severely inhibited ISG and $\beta$-IFN expression, clearly demonstrating the involvement of these transcription factors in the triggering of the antiviral response. However, further examination of the pattern recognition receptors (PRR) potentially involved in the induction of this response demonstrated that neither inhibition of endosome-resident TLR signalling by blocking lysosome maturation with ammonium chloride nor downregulation of TLR3 and TLR4 using specific siRNA, were able to abolish the cellular response to amplicons. This indicates that none of the TLR that are generally associated with IFN responses actually participates to the induction of this cellular response. Moreover, preliminary results from the same laboratory suggest that the induction of the innate response in HFFF-2 cells is also independent of some of the cytosolic recognition receptors, such as RIG-I or DAI, which have been described as sensors of cytosolic RNA and DNA. Other cytosolic receptors of nucleic acids have however being recently identified, but they have not yet been tested for amplicon recognition. These findings raise the pertinent question of how these cells recognize that they have been infected by amplicons. Most interestingly, although amplicon-mediated transgene expression is extremely weak in HFFF-2 cells, down-regulation of the induced innate response with IRF3 and IRF7 specific siRNA did not enhance transgene expression, suggesting that the low-level expression observed cannot be solely explained by the induction of the cellular innate response [40].

Taken together, the above-described investigations clearly demonstrate that HF-amplicon infection can elicit innate immune and inflammatory responses, mediated by IRFs (IFN) and by NF- $\kappa \mathrm{B}$ (inflammatory cytokines), both in cultured cells and in vivo. While these molecules certainly play a major role in the induction of adaptive immune responses in the inoculated organisms, its role in the silencing of transgene expression at the cellular level is not clearly established, suggesting that other cellular silencing forces could be at work. It is important to stress that the IRF3/7- and NF- $\kappa \mathrm{B}-$ mediated responses are transient and that cells will rapidly resume their normal activities and gene expression patterns. On the other hand, although it has been shown that HSV-1 could induce (and inhibit) apoptotic responses in the infected cells $[1,10,11]$, induction of apoptosis requires expression of virus genes that are normally absent from HF-amplicons. These vectors therefore do not induce apoptosis and are absolutely non-toxic for the transduced cells.

\section{CONSTITUTIVE ANTIVIRAL RESPONSES CAN INHIBIT AMPLICON-MEDIATED GENE EXPRESSION}

In recent years it has became evident that a group of constitutively expressed proteins could also be involved in the negative regulation of HSV-1 gene expression [41-44]. These molecules, which include ND10-associated proteins, such as PML, sp100, ATRX or hDaxx [41-43], as well as ND10-independent chromatin associated proteins, such as the Rest-Corest-HDAC co-repressor system [44], seems to act mainly as transcriptional repressors. Although these molecules are constitutively expressed, at least some of them, including PML and sp100, contain interferon- stimulated response elements (ISRE) in their regulatory sequences and can be further induced by $\beta$-IFN, thus establishing a connection between these transcriptional silencers and the IFN-mediated system [45]. Some of these molecules can, however, repress expression of the incoming HSV-1 genome even in the absence of IFN, particularly in human fibroblasts, and this action can be counteracted by HSV-1 encoded proteins, and particularly by ICP0 [41-44].

In a study conceived to investigate how amplicon vectors function in vivo to generate an immune response, amplicon vectors expressing either GFP or luciferase reporter proteins were used to examine the duration of expression after administration to mice [46]. Injection with amplicons expressing luciferase revealed that transgene expression peaked earlier than $24 \mathrm{hr}$ after injection in mice. With amplicons expressing GFP, transgene expression peaked at $24 \mathrm{hr}$ following in vivo administration. Thus, both reporter proteins revealed a rather rapid and robust expression pattern of short duration, which appears in part to be due to gene silencing. Examination of cells displaying transgene expression suggested that amplicons could transduce a variety of cells, including professional antigen-presenting cells. Then, a very interesting study, looking at potential reasons underlying the observed transgene repression, indicated that bacterial sequences present in the amplicon genome could cause rapid transgene transcriptional silencing by forming inactive chromatin [47]. Indeed, each amplicon vector genome contains several copies of the original plasmid sequence, depending on the size of the amplicon plasmid [20]. Infection with amplicons devoid of bacterial sequences (minicircle amplicons) induced approximately 20fold higher transgene expression than conventional amplicons, and quantitative analysis of levels of transgenic mRNA revealed that the increase in transgene expression was at the transcriptional level. In addition, nude mice injected with minicircle amplicons exhibited 10-fold higher luciferase expression than mice injected with conventional amplicons. Furthermore, luciferase expression from conventional amplicons was undetectable 21 days after injection, whereas with the minicircle amplicons expression was detectable up to at least 28 days post-infection. These observations suggest that inherently expressed cellular proteins, perhaps recognizing naked foreign DNA entering the nucleus, are probably implicated in the silencing of the amplicon genome [47].

Other studies aimed at investigating a possible role for the viral protein ICP0 in the inhibition of cellular silencing. ICP0 is an HSV-1 immediate early regulatory protein that plays a major role in virus infection by activating all classes of virus gene expression [48]. Everett and co-workers, and other groups, have shown that ICP0-null HSV-1 mutants can infect human fibroblasts and deliver the virus genome to the cell nucleus, but expression from the virus genome is immediately silenced when the cells are infected at low multiplicity, at least in part due to the silencing action of PML and other ND10-related proteins [41-43]. The immediate-early ICP0 protein, which contains an E3ubiquitin ligase activity, allows overcoming this repression by inducing the proteasome-dependent proteolytic degradation of several ND10-related proteins [48]. Since amplicons express neither ICP0 nor any other viral genes, it is possible that the very low level of transgenic expression 
observed in human fibroblasts and other cell types could be caused by the action of these inherent cellular silencing responses. In support of such hypothesis, it was observed that amplicons simultaneously expressing GFP under the control of the IE4/5 HSV-1 promoter, and wild-type ICP0 driven by the HCMV promoter, expressed higher levels of GFP in human primary fibroblasts, cultured rat cardiomyocytes and rat neonatal cultured brain cells, than amplicons expressing no ICP0 or a mutated inactive form of ICP0 (FXE), suggesting that ICP0 played a significant role in suppressing a silencer activity in the amplicon-infected cells [21]. Since, as already quoted, one important role of ICP0 is to induce degradation of several ND10 constitutive proteins, these results lend further support to the hypothesis that an inherent antiviral mechanism mediated by these proteins is partially responsible for the silencing of amplicon-mediated transgene expression in human fibroblasts. Furthermore, preliminary results from our laboratory indicate that siRNA down-regulation of Sp100 and Daxx, two regulatory proteins that localize to ND10 bodies, resulted in at least 5 -fold enhancement in the number of GFP-expressing normal human fibroblasts infected with standard amplicons [40].

A very recent study, also intended to test the hypothesis that expression of ICP0 could overcome silencing of encoded transgenes, used amplicons expressing ICP0 under the control of its native promoter [49]. Expression of ICP0 from these amplicons was transient and did not significantly alter IFN-based responses against the vector or cell kinetics/apoptosis of infected cells. Chromatin immunoprecipitation (ChIP)/PCR analysis revealed that conventional amplicon DNA became associated with HDAC1, a protein associated with transcriptional repression, immediately after infection. In contrast, with ICP0expressing amplicons the vector DNA remained relatively unbound by $\mathrm{HDACl}$ for at least $72 \mathrm{hr}$ post-infection. In addition, mice systemically inoculated with amplicons expressing ICP0 exhibited significantly greater and more sustained transgene expression in their livers than did those receiving conventional amplicons. Thus, these results add further support to the notion that amplicon expression is submitted to silencing and that ICP0 could restore normal expression or at least decrease transgene silencing.

Lastly, the observation that the reduction of particleassociated ICP0 levels, which results from packaging the amplicon genome in the presence of the transcriptional regulator hexamethylene bisacetylamide (HMBA), also results in reduced transgene expression, provides further additional evidence for a role of tegumentary ICP0 in suppressing the silencing mechanisms that repress ampliconmediated transgene expression [50].

Taken together, the above-described results clearly indicate that cellular silencing forces can act on the amplicon genome to block transgene expression and that these forces could be disabled by ICP0 or by external intervention aimed at suppressing them, such as RNAi procedures.

\section{CONCLUDING REMARKS: TOWARDS STABLE AMPLICON-MEDIATED TRANSGENE EXPRESSION}

Results and observations described in this short review indicate that at least two types of cellular protective mechanisms are acting in cells infected with HF-amplicon vectors. A first one is mediated by constitutively expressed proteins that can target the incoming vector genome as soon as it enters the nucleus of the infected cells. The experimental evidence seems to support the hypothesis that at least two different silencing mechanisms mediated either by ND10-associated proteins or by HDAC1-associated proteins, both of which are targeted to degradation by ICP0, could play a major role in silencing the vector genome. In addition, a large set of observations sustain the notion that HF-amplicons could induce type I IFN-mediated and NF$\kappa \mathrm{B}$-mediated innate immune responses, at least in some experimental settings. It is clear that the induction of this innate responses will play a role in activating the adaptive branch of immunity. However, the evidence favouring a role of these responses in the control of transgene expression at the cellular level is not yet conclusive. While activation of STAT1 in mice resulted in decreased vector expression, therefore suggesting that antiviral mechanisms elicited by type I IFN can control amplicon expression, in cultured human fibroblasts, down-regulation of IRF3 and 7 did not enhanced transgene expression. Although apparently contradictory, these experiments actually address different moments of the innate response and could be reconciled by the fact that, in cultured fibroblasts, the level of induced and secreted type I IFN was quite low, perhaps not high enough as to elicit the full set of antiviral proteins. More work is therefore required to evaluate the part played by type I IFN responses in the inhibition of amplicon-mediated transgene expression.

For gene therapy purposes, the critical point is if it would be possible to generate rationally designed amplicon vectors able to avoid gene silencing, therefore leading to stable transgene expression. A possible approach to this end is based on the demonstration that bacterial DNA-free amplicons are better armed to escape silencing due to histone deposition than conventional amplicons [47]. It is possible that other not yet identified sequences in the vector genome could also be involved in silencing. Identification and deletion of these sequences, if any, would also have a positive impact in expression. Alternatively, or in addition, it would be certainly possible to engineer the amplicon genome in order to express functions potentially able to inhibit cellular-induced gene silencing without toxicity for the transduced cells, or to include tissue-specific regulatory DNA elements, thereby forcing the amplicon genome to generate a transcriptionally active chromatin configuration, thus allowing stable expression. Previous observations using tissue-specific promoters or enhancers actually support the great interest of this approach [27-30].

A different strategy would consist in the engineering the helper system used to generate the vectors, aiming at modifying the protein content of the amplicon particles. Using such approach, it is possible to speculate on the possibility of modifying the tegument or the envelope of the amplicon particle, in order to introduce proteins that could act on- and block- constitutive or inducible silencing systems. These approaches seems today far from reality, but they are not. The possibility of using helper virus genomes carried by bacterial artificial chromosomes, together with the improvement in our ability to engineer this molecule through homologous or site-specific recombination in bacteria, has 
already proven useful to modify recombinant HSV-1 vectors [51] and will certainly help to efficiently screen, identify, and modify, the virus genes that should be engineered in order to reach these ends.

\section{ACKNOWLEDGEMENTS}

We are grateful to the French societies Association pour la Recherche contre le Cancer (ARC) and Association Française contre les Myopathies (AFM) for constant support to our laboratory.

\section{REFERENCES}

[1] Leopardi R, Van Sant C, Roizman B. The herpes simplex virus 1 protein kinase Us3 is required for protection from apoptosis induced by the virus. Proc Natl Acad Sci USA 1997; 94: 7891-6.

[2] Peri P, Mattila RK, Kantola H, et al. Herpes simplex virus type 1 US3 gene délétion influences Toll-like receptor responses in culture monocytic cells. Virol J 2008; 5: 140-51.

[3] Mossman KL, Smiley JR. Herpes simplex virus ICP0 and ICP34.5 counteract distinct interferon-induced barriers to virus replication. J Virol 2002; 76: 1995-8.

[4] Eidson KM, Hobbs WH, Manning BJ, Carlson P, DeLuca NA. Expression of herpes simplex virus ICP0 inhibits the induction of interferon-stimulated genes by viral infection. J Virol 2002; 76: 2180-91.

[5] Cheng G, Brett ME, He B. Val193 and Phe195 of the $\gamma 134.5$ Protein of Herpes Simplex Virus 1 Are Required for Viral Resistance to Interferon- $\alpha / \beta$. Virology $2001 ; 290: 115-20$.

[6] Orvedahl A, Alexander D, Talloczy Z, et al. HSV-1 ICP34.5 confers neurovirulence by targeting the Beclin 1 autophagy protein. Cell Host Microbe 2007; 1: 23-35.

[7] Duerst RJ, Morrison LA. Herpes simplex virus 2 virion host shutoff protein interferes with type I interferon production and responsiveness. Virology 2004; 322: 158-67.

[8] Johnson KE, Song B, Knipe DM. Role for herpes simplex virus 1 ICP27 in the inhibition of type I interferon signaling. Virology 2008; 374: 487-94.

[9] York IA, Roop C, Andrews DW, et al. A cytosolic herpes simplex virus protein inhibits antigen presentation to $\mathrm{CD} 8+\mathrm{T}$ lymphocytes. Cell 1994; 77: 525-35.

[10] Jerome KR, Chen Z, Lang R, et al. HSV and glycoprotein J inhibit caspase activation and apoptosis induced by granzyme B or fas. $\mathrm{J}$ Immunol 2001; 167: 3928-35.

[11] Zhou G, Galvan V, Campadelli-Fiume G, Roizman B. Glycoprotein D or J delivered in trans blocks apoptosis in SK-NSH cells induced by a herpes simplex virus 1 mutant lacking intact genes expressing both glycoproteins. J Virol 2000; 74: 11782-91.

[12] Mulvey M, Camarena V, Mohr I. Full resistance of herpes simplex virus type 1-infected primary human cells to alpha interferon requires both the Us11 and $\gamma 134.5$ gene products. J Virol 2004; 78: 10193-6.

[13] Dubin G, Socolof E, Franck I, Friedman HM. Herpes simplex virus type $1 \mathrm{Fc}$ receptor protects infected cells from antibody-dependent cellular cytotoxicity. J Virol 1991; 65: 7046-50.

[14] Friedman HM, Cohen GH, Eisenberg RJ, Seidel CA, Cines DB. Glycoprotein $\mathrm{C}$ of herpes simplex virus 1 acts as a receptor for the C3b complement component on infected cells. Nature 1984; 309: 633-5.

[15] Kostavasili I, Sahu A, Friedman HM, Eisemberg RJ, Cohen GH, Lambris JD. Mechanism of complement inactivation by glycoprotein C of herpes simplex virus. J Immunol 1997; 158: 1763-71.

[16] Leopardi R, Roizman B. The herpes simplex virus major regulatory protein ICP4 blocks apoptosis induced by the virus or by hyperthermia. Proc Natl Acad Sci USA 1996; 93: 9583-7.

[17] Krisky DM, Marconi PC, Oligino TJ, et al. Development of herpes simplex virus replication-defective multigene vectors for combination gene therapy applications. Gene Ther 1998; 5: 151730.

[18] Wu N, Watkins SC, Schaffer PA, DeLuca NA. Prolonged gene expression and cell survival after infection by a herpes simplex virus mutant defective in the immediate-early genes encoding ICP4, ICP27, and ICP22. J Virol 1996; 70: 6358-69.
[19] Spaete RR, Frenkel N. The herpes simplex virus amplicon: a new eucaryotic defective-virus cloning-amplifying vector. Cell 1982; 30: 295-304

[20] Cuchet D, Potel C, Thomas J, Epstein AL. HSV-1 amplicon vectors: a promising and versatile tool for gene delivery. Exp Opin Biol Ther 2007; 7: 975-95.

[21] Cuchet D, Ferrera R, Lomonte P, Epstein, AL. Characterization of antiproliferative and cytotoxic properties of the HSV-1 immediateearly ICP0 protein. J Gene Med 2005; 7: 1187-99.

[22] During MJ, Naegele JR, O'Malley KL, Geller AI. Long-term behavioral recovery in parkinsonian rats by an HSV vector expressing tyrosine hydroxylase. Science 1994; 266: 1399-403.

[23] Fraefel C, Song S, Lim F, et al. Helper virus-free transfer of herpes simplex virus type 1 plasmid vectors into neural cells. J Virol 1996; 70: 7190-7.

[24] Saeki Y, Ichikawa T, Saeki A, et al. Herpes simplex virus type 1 DNA amplified as bacterial artificial chromosome in Escherichia coli: rescue of replication-competent virus progeny and packaging of amplicon vectors. Hum Gene Ther 1998; 9: 2787-94.

[25] Saeki Y, Fraefel C, Ichikawa T, Breakefield XO, Chiocca EA Improved helper virus-free packaging system for HSV amplicon vectors using an ICP27-deleted, oversized HSV-1 DNA in a bacterial artificial chromosome. Mol Ther 2001; 3: 591-601.

[26] Zaupa C, Revol-Guyot V, Epstein AL. Improved packaging system for generation of high-level noncytotoxic HSV-1 amplicon vectors using Cre-loxP site-specific recombination to delete the packaging signals of defective helper genomes. Hum Gene Ther 2003; 14: 1049-63.

[27] Wade-Martins R, Smith ER, Tyminski E, Chiocca EA, Saeki Y. An infectious transfer and expression system for genomic DNA loci in human and mouse cells. Nat Biotechnol 2001; 19: 1067-70.

[28] Wade-Martins R, Saeki Y, Chiocca EA. Infectious delivery of a 135-kb LDLR genomic locus leads to regulated complementation of low-density lipoprotein receptor deficiency in human cells. Mol Ther 2003; 7: 604-12.

[29] Moralli D, Simpson KM, Wade-Martins R, Monaco ZL. A novel human artificial chromosome gene expression system using herpes simplex virus type 1 vectors. EMBO Rep 2006; 7: 911-8.

[30] Gomez-Sebastian S, Gimenez-Cassina A, Diaz-Nido J, Lim F, Wade-Martins R. Infectious delivery and expression of a $135 \mathrm{~kb}$ human FRDA genomic DNA locus complements Friedreich's Ataxia Deficiency in human cells. Mol Ther 2007; 15: 248-54.

[31] Hodge PB, Stow ND. Effects of mutations within the herpes simplex virus type 1 DNA encapsidation signal on packaging efficiency. J Virol 2001; 75: 8977-86.

[32] Sourvinos G, Everett RD. Visualization of parental HSV-1 genomes and replication compartments in association with ND10 in live infected cells. EMBO J 2002; 21: 4989-97.

[33] Porter IM, Stow ND. Replication, recombination and packaging of amplicon DNA in cells infected with the herpes simplex virus type 1 alkaline nuclease null mutant ambUL12. J Gen Virol 2004; 85: 3501-10.

[34] Tsitoura E, Thomas J, Cuchet D, Thoinet K, Mavromara P, Epstein AL. Infection with herpes simplex type 1-based amplicon vectors results in an IRF3/7-dependent, TRL-independent activation of the innate antiviral response in primary human fibroblasts. J Gen Virol 2009; 90: 2209-20.

[35] Tolba KA, Bowers WJ, Hilchey SP, et al. Development of herpes simlex virus-1 amplicon-based immunotherapy for chronic lymphocytic leukemia. Blood 2001;98: 287-95.

[36] Daubeuf S, Singh D, Tan Y, et al. HSV ICP0 recruits USP7 to modulate TLR-mediated innate response. Blood 2009; 113: 326475.

[37] Olschowka JA, Bowers WJ, Hurley SD, Mastrangelo MA, Federoff HJ. Helper-free HSV-1 amplicons elicit a markedly less robust innate immune response in the CNS. Mol Ther 2003; 7: 218-27.

[38] Suzuki M, Chiocca EA, Saeki Y. Early STAT1 activation after systemic delivery of HSV amplicon vectors suppresses transcription of the vector-encoded transgene. Mol Ther 2007; 15: 2017-26.

[39] Suzuki M, Chiocca EA, Saeki Y. Stable transgene expression from HSV amplicon vectors in the brain: potential involvement of immunoregulatory signals. Mol Ther 2008; 16: 1727-36.

[40] Tsitoura E, Epstein AL. Personal communication. Preliminary results were presented at the 31th International Herpesvirus 
Workshop, Seattle, USA (2006) and at the 14th Meeting of the European Society of Gene Therapy, Athens, Greece (2006).

[41] Everett RD, Boutell C, Orr A. Phenotype of a herpes simplex virus type 1 mutant that fails to express immediate-early regulatory protein ICP0. J Virol 2004; 78: 1763-74.

[42] Everett RD, Rechter S, Papior P, et al. PML contributes to a cellular mechanism of repression of herpes simplex virus type 1 infection that is inactivated by ICP0. J Virol 2006; 80: 7995-8005.

[43] Everett RD, Parada C, Gripon P, Sirma H, Orr A. Replication of ICP0-null mutant herpes simplex virus type 1 is restricted by both PML and Sp100. J Virol 2008; 82: 2661-72.

[44] Gu H, Liang Y, Mandel G, Roizman B. Components of the REST/CoREST/histone deacetylase repressor complex are disrupted, modified, and translocated in HSV-1-infected cells. Proc Natl Acad Sci USA 2005; 102: 7571-6.

[45] Chelbi-Alix MK, de Thé H. Herpes virus induced proteasomedependent degradation of the nuclear bodies-associated PML and Sp100 proteins. Oncogene 1999; 18: 935-41.

[46] Santos K, Simon DA, Conway E, et al. Spatial and temporal expression of herpes simplex virus type 1 amplicon-encoded genes: implications for their use as immunization vectors. Hum Gene Ther 2007; 18: 93-105.

[47] Suzuki M, Kasai K, Saeki Y. Plasmid DNA sequences present in conventional herpes simplex virus amplicon vectors cause rapid transgene silencing by forming inactive chromatin. J Virol 2006; 80: 3293-300

[48] Everett RD. ICP0, a regulator of herpes simplex virus during lytic and latent infection. BioEssays 2000; 22: 761-70.

[49] Suzuki M, Kasai K, Ohtsuki A, et al. ICP0 inhibits the decrease of HSV amplicon-mediated transgene expresion. Mol Ther 2009; 17: 707-12.

[50] Burris CA, De Silva S, Narrow WC, et al. Hexamethylene bisacetamide leads to reduced helper virus-free HSV-1 amplicon expression titers via suppression of ICP0. J Gene Med 2008; 10: $152-64$.

[51] de Oliveira AP, Glauser DL, Laimbacher A, et al. Live visualisation of herpes simplex virus type 1 compartment dynamics. J Virol 2008; 82: 4974-90.

[52] Favoreel HW, Van de Walle GR, Nauwynck HK, Pensaert MB. Virus complement evasion strategies. J Gen Virol 2003; 84: 1-15.

[53] Frank I, Friedman HM. A novel function of the herpes simplex virus type $1 \mathrm{Fc}$ receptor: participation in bipolar bridging of antiviral immunoglobulin G. J Virol 1989; 63: 4479-88.

[54] Lin R, Noyce RS, Collins SE, Everett RD, Mossman KL. The herpes simplex virus ICP0 RING finger domain inhibits IRF3- and IRF7-mediated activation of interferon-stimulated genes. J Virol 2001; 78: 1675-84.

[55] Halford WP, Weisend C, Grace J, et al. ICP0 antagonizes Stat1dependent repression of herpes simplex virus: implications for the regulation of viral latency. Virol J 2006; 3: 44.

[56] Chee AV, Lopez P, Pandolfi PP, Roizman B. Promyelicytic leukemia protein mediates interferon-based anti-herpes simplex virus effects. J Virol 2003; 77: 7101-5.

[57] Melchjorsen J, Sirén J, Julkunen I, Paludan SR, Matikainen S. Induction of cytokine expression by herpes simpelx virus in human monocyte-derived macrophages and dendritic cells is dependent on virus replication and is counteracted by ICP27 targeting NFkappaB and IRF3. J Gen Virol 2006; 87: 1099-108.

[58] He B, Gross M, Roizman B. The gamma(1)34.5 protein of herpes simplex virus 1 complexes with protein phosphatase 1alpha to dephosphorylate the alpha subunit of the eukaryotic translation initiation factor 2 and preclude the shutoff of protein synthesis by double-stranded RNA-activated protein kinase. Proc Natl Acad Sci USA 1997; 94: 843-8.

[59] Verpooten D, Ma Y, Hou S, Yan Z, He B. Control of TANKbinding kinase 1-mediated signaling by the $\gamma 134.5$ protein of herpes simplex virus 1. J Biol Chem 2008; 284: 1097-105.

[60] Sanchez R, Mohr I. Inhibition of cellular 2'-5' oligoadenylate synthetase by the herpes simplex virus type 1 Us11. J Virol 2007; 81: 3455-464.

[61] Cassady KA, Gross M, Roizman B. The herpes simplex virus Us11 protein effectively compensates for the gamma1(34.5) gene if present before activation of protein kinase $\mathrm{R}$ by precluding its phosphorylation and that of the alpha subunit of eukaryotic translation initiation factor 2. J Virol 1998; 72: 8620-6.

[62] Eisemann J, Mühl-Zurbes P, Steinkasserer A, Kummer M. Infection of mature dendritic cells with herpes simplex virus type 1 interferes with the interferon signaling pathway. Immunobiology 2007; 212: 877-86.

[63] Liang L, Roizman B. Expression of gamma interferon-dependent genes is blocked independently by virion host shutoff RNAse and bu Us3 protein kinase. J Virol 2008; 82: 4688-96.

[64] Hagglund R, Munger J, Poon AP, Roizman B. U(S)3 protein kinase of herpes simplex virus 1 blocks caspase 3 activation induced by the products of $\mathrm{U}(\mathrm{S}) 1.5$ and $\mathrm{U}(\mathrm{L}) 13$ genes and modulates expression of transduced $\mathrm{U}(\mathrm{S}) 1.5$ open reading frame in a cell typespecific manner. J Virol 2002; 76: 743-54.

[65] Benetti L, Munger J, Roizman B. The herpes simplex virus 1 US3 protein kinase blocks caspase-dependent double cleavage and activation of the proapoptotic protein BAD. J Virol 2003; 77: 656773.

[66] Munger J, Roizman B. The US3 protein kinase of herpes simplex virus 1 mediates the posttranslational modification of $\mathrm{BAD}$ and prevents BAD-induced programmed cell death in the absence of other viral proteins. Proc Natl Acad Sci USA 2001; 98: 10410-5.

[67] Ogg PD, McDonell PJ, Ryckman BJ, Knudson CM, Roller R. The HSV-1 US3 protein kinase is sufficient to block apoptosis induced by overexpression of a variety of Bcl-2 family members. Virology 2004; 319: 212-24.

[68] Aubert M, Blaho JA. Viral oncoapoptosis of human tumor cells Gene Ther 2003; 10: 1437-45.

[69] Nguyen ML, Kraft RM, Blaho JA. African green monkey kidney Vero cells require de novo protein synthesis for efficient herpes simplex virus 1-dependent apoptosis. Virology 2005; 336: 274-90.

[70] Aubert M, Blaho JA. The herpes simplex virus type 1 regulatory protein ICP27 is required for the prevention of apoptosis in infected human cells. J Virol 1999; 73: 2803-13.

[71] Gregory D, Hargett D, Holmes D, Money E, Bachenheimer SL. Efficient replication by herpes simplex virus type 1 involves activation of the IkappaB kinase-IkappaB-p65 pathway. J Virol 2004; 78: 13582-90.

[72] Medici MA, Sciortino MT, Perri D, et al. Protection by herpes simplex virus glycoprotein D against Fas-mediated apoptosis; role of nuclear factor kappaB. J Biol Chem 2003; 278: 36059-67.

[73] Perng GC, Jones C, Stone M, et al. Virus-induced neuronal apoptosis blocked by the herpes simplex virus latency-associated transcript. Science 2000; 287: 1500-3.

[74] Henderson G, Peng W, Jin L, et al. Regulation of caspase 8- and caspase 9-induced apoptosis by the herpes simplex virus type 1 latency-associated transcript. J Neurovirol 2002; 8(S2): 103-11. 